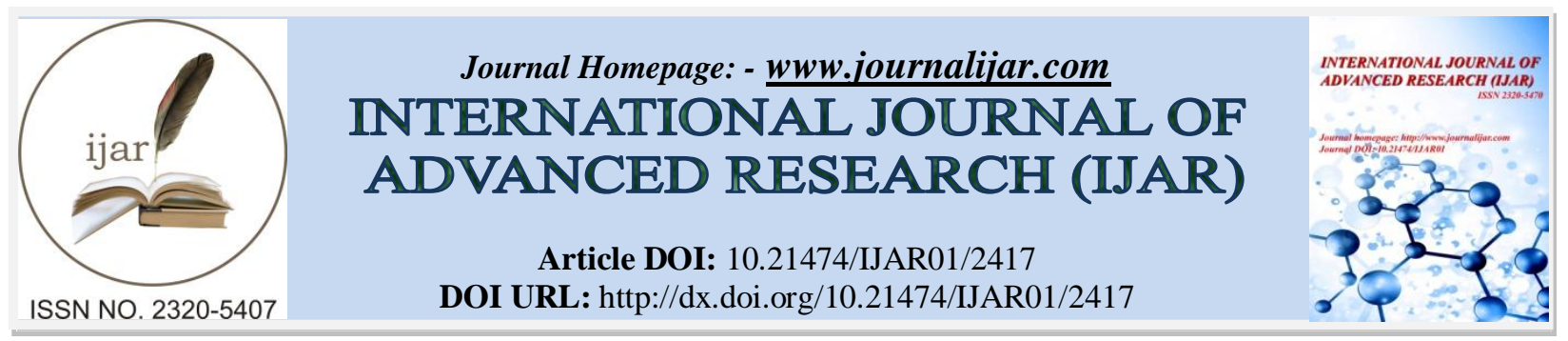

RESEARCH ARTICLE

\title{
BLOOD PRESSURE METER USING NEAR-INFRARED SPECTROSCOPY SENSORS.
}

\section{Dr. U. Suneetha ${ }^{1}$ and Prof.B.Rama Murthy ${ }^{2}$.}

1. Department of Electronics, S.K.University, Anantapur, A.P.

2. Department of Instrumentation, S.K.University, Anantapur, A.P.

\section{Manuscript Info}

Manuscript History

Received: 20 October 2016

Final Accepted: 22 November 2016

Published: December 2016

Key words:-

Near-infrared spectroscopy, Infrared

light, micro controller, blood pressure

\section{Abstract}

A blood pressure meter is designed by using near-infrared spectroscopy (NIRS). Blood pressure estimations are find by recording pulse wave velocity of blood traveling from the upper arm down to one's index finger. The heart contracts and relaxes, it pumps blood through the body, creating the pulse felt at different parts of the body. This pulse is a brief increase in blood flow that occurs continuously and can be detected as a periodic signal. Because NIRS can detect the amount of blood flow, when the pulse wave arrives at a certain point in the body, the amount of IR light reflected back out through the skin increases. The signal output from the IR sensor is therefore the pulse signal itself, if filtered correctly. Near-infrared spectroscopy (NIRS) is chose to measuring blood pressure through collecting data on pulse transit time between two points in the body. Read in data through two analog inputs on the controller, and calculate the time at which the pulse wave reached each location mentioned.

Copy Right, IJAR, 2016,. All rights reserved.

\section{Introduction:-}

Blood pressure (BP) is an importantfactor to examine physical attributes and it is useful to indicate cardiovascular diseases, so the measurement of blood pressure has gained increasing attention. Therefore, BP is an important indicator for health monitoring [1]. Conventional methods of BP monitoring are divided into direct and indirect ways. In direct way, the pressure sensor must be implanted in artery and can continuously monitor BP, but the operation is complicated and difficultly acceptable for patients. The indirect way is the mercury sphygmomanometer, the current gold standard for BP measurement [2].

Near infrared spectroscopy has numerous applications in clinical settings, due to its effectiveness and the fact that it is non-invasive. At certain wavelengths infrared light is able to penetrate human skin and bone. An IR LED can be oriented on the skin such that it is shining light into the arm. When the IR light reaches blood vessels in the human body, it reflects off hemoglobin in the blood [3]. Some of this reflected light scatters in random directions, but a good amount of the light is reflected back out through the skin. If an IR light detector is located on the skin next to the IR LED, also facing into the skin, this sensor can detect the amount of light reflected back out.

As the heart contracts and relaxes, it pumps blood through the body, creating the pulse felt at different parts of the body. This pulse is a short-term increase in blood flow that occurs continuously, and therefore can be detected as a periodic signal. Because NIRS can detect the amount of blood flow, when the pulse wave arrives at a certain point in the body, the amount of IR light reflected back out through the skin increases. The signal output from the IR sensor 
is therefore the pulse signal itself, if filtered correctly. The present work is divided in to different blocks those are present in below.

\section{Design:-}

The design procedure split into following stages, those are

1. The IR sensor,

2. Filtering stages

3. Voltage offset circuit

4. Atmega8 micro controller

The block diagram of Blood pressure measurement is shown in figure 1.

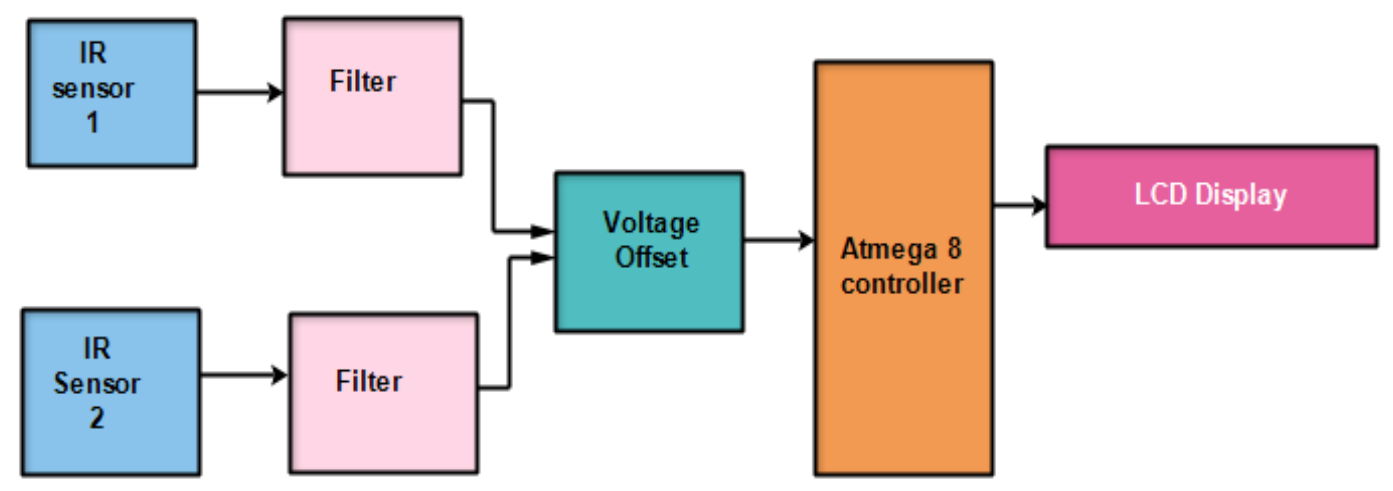

Figure 1:- Block diagram for blood pressure measurement near infrared spectroscopy

\section{IR Sensor:-}

IR sensor is made up of an IR LED emitting light at $940 \mathrm{~nm}$ into the skin, as well as an IR light sensor. A photodiode is used as light sensor. We chose this component rather than a regular IR detector because the OPT101 has a trans impedance amplifier on board, converting the current to voltage and amplifying the output. These two components, the LED and sensor, were soldered onto a small board, both facing the same direction and with a little space between them so that light reflected back out scatters such that its highest intensity is not directly next to from where it was emitted. The photodiode is also powered by $5 \mathrm{~V}$ on the breadboard, and also includes a $0.1 \mu \mathrm{F}$ decoupling capacitor between its power and ground lines. The output of the photodiode goes to the input of the filters.

\section{Filters:-}

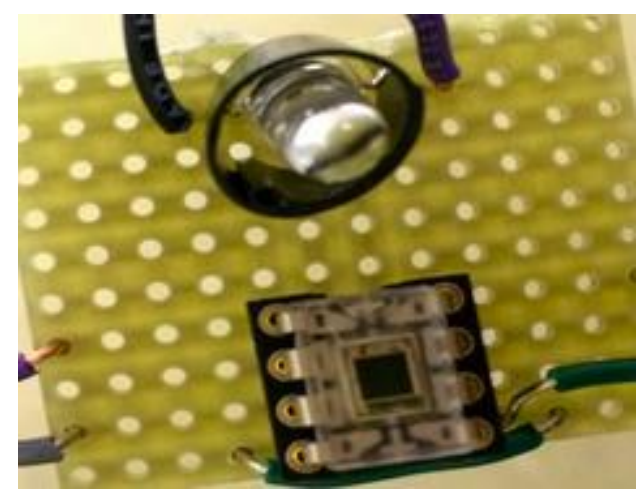

Photograph1: IR Sensor PCB photograph.

The filter stage consists of a high pass filter and low pass filter, with some amplification. Designing the filters bandwidth approximately $0.1 \mathrm{~Hz}$ to $10 \mathrm{~Hz}$ in order to capture all components of the pulse wave, while filtering out high frequency noise. The two filters were combined into a non-inverting operational amplifier (LM2904) circuit, which also included gain of about 34 . For the low pass filter, a $0.47 \mu \mathrm{F}$ capacitor and a $33 \mathrm{k} \Omega$ resistor to get the cutoff frequency of $10 \mathrm{~Hz}$. For the high pass filter, a $10 \mu \mathrm{F}$ capacitor and a $160 \mathrm{k} \Omega$ resistor to get the cut-off frequency of 
$0.1 \mathrm{~Hz}$. There is also a $1 \mathrm{k} \Omega$ resistor going into the negative input of the op-amp to ground in order to create a gain of 34 together with the $33 \mathrm{k} \Omega$ feedback resistor. The output of the sensor goes into the non-inverting input on the opamp. At the output of the op-amp, a small pulse signal can be seen, approximately centered around 0V.

\section{Voltage offset circuit:-}

The output of the previous filter stage goes into the non-inverting input on an op-amp (MCP6241). The persistence of this circuit is to shift the $+/-5 \mathrm{~V}$ signal from the previous output to $0 \mathrm{~V}$ to $3.3 \mathrm{~V}$ before going into the Atmega8. The op-amp circuit is configured as a differential amplifier, with the input going into the positive input on the opamp, and a voltage offset going into the negative input. The gain is set by a feedback resistor, and the pairs of resistors are matched in order to have a common mode gain of zero. The op-amp was powered by $0 \mathrm{~V}$ and $3.3 \mathrm{~V}$ in order to set the maximum and minimum barriers of our signal. In order to produce the voltage offset, a $10 \mathrm{k} \Omega$ potentiometer between $-5 \mathrm{~V}$ and $0 \mathrm{~V}$ to create an adjustable voltage divider. The offset ended up being roughly $1.6 \mathrm{~V}$. Finally, the gain set in this stage differed between the arm sensor circuit and the finger sensor circuit. These two places on our body obviously have physical differences, and the signal given from the sensor varies between them. Therefore, we needed to set the gain differently for each. After being amplified and shifted, the pulse signal at the output of this circuit was now sufficient to be input to the ATMEGA8.

\section{Atmega8 micro controller:-}

The program is to configure our Atmega8 follows a relatively simple structure. It begins by setting up the 10-bit analog-to-digital converter and it is configured such that it has the capability to read from two analog inputs. When all the samples have been collected, the program a value for blood pressure has been calculated at this point, it will display on the LCD otherwise it will display a message informing the user that calibration data is still being collected (different than the data collected for each iteration of the data arrays). Finally, the ADC thread spawns the blood pressure calculation thread.

The two data arrays are analyzed in order to calculate systolic blood pressure. As described previously, blood pressure can be calculated using the difference in the arrival times of two pulse waveforms measured at two different places on the body. In order to determine this time difference, we chose to measure the time between the voltage midpoints of each pulse wave $[4,5]$. We chose the midpoint rather than the minimum and maximum because of the DC drift that the pulse waveform undergoes. The first task this thread has is to determine the maximum and minimum voltages for each of the two pulse waveforms, from which midpoint can be found. The program iterates through each data vector to find and max and min voltage values, and also saves the indices of them. The midpoint value for each data set is calculated as halfway between the min and max values.

Once the program has index values for the min, max, and midpoint for each dataset, it can calculate the lag in time between the two waveforms, which is the same as the difference in arrival times of the two pulse waveforms. The algorithm we wrote for finding the midpoint of each waveform, while most of the time correct, sometimes detected the wrong midpoint value and/or index. We define a "correct" midpoint as it being between the min and max values in time, for both pulse datasets. If the value/index is incorrect, we discard it and do not use it in our blood pressure calculation. If both are determined to be correct, the lag time in seconds is calculated and saved to a vector of lag times. Lag time is used in determining blood pressure, but rather than using each lag time to calculate a blood pressure value, we decided to collect 50 samples of lag times, which are averaged. It is this average lag time that is used to calculate blood pressure. This is one aspect of calibration we realized was necessary, as using a single lag time to calculate blood pressure resulted in an erroneous value. The complete schematic diagram is shown in figure 2. 


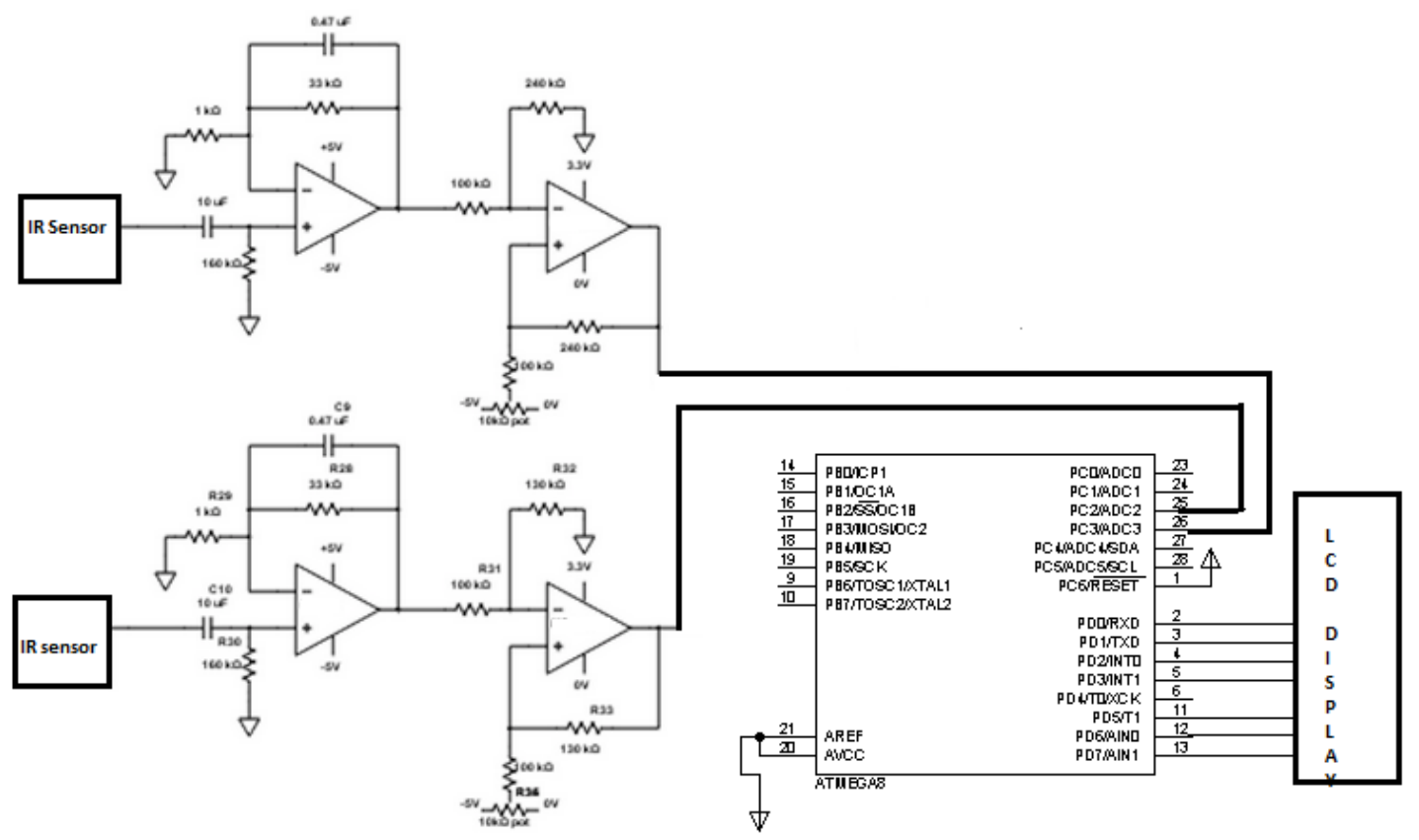

Figure 2:- schematic for blood pressure measurement using near infrared Sensors.

\section{LCD Display:-}

The LCD Display Module we used to display data is 4×20 characters. LCD display it has a great contrast and a wide viewing angle. Display module is controlled by SPLC780D parallel interface chipset that is easy to use.

\section{Results:-}

The final results successfully met our expectations. Our design is accurate which is matched with the regularly used device with small error. Our blood pressure monitor varies from person to person as well as within one individual, due to various physiologic characteristics.

\section{References:-}

1. Wong MY, Poon CC, Zhang YT. An evaluation of cuffless blood pressure estimation based on pulse transit time technique: a half study on normotensive subjects. Cardiovasc Eng. 2009 Mar;9(1):32-8.

2. Sheng H, Schwarz M, Boercsoek J. A wireless wearable body sensor network for continuous noninvasive blood pressure monitoring using multiple parameters. Proceedings of the 2nd international conference on Circuits, Systems, Communications \& Computers; Puerto De La Cruz, Tenerife, Spain. 2209499: World Scientific and Engineering Academy and Society (WSEAS); 2011. p. 308-14.

3. Continuous Blood Pressure Determination during Exercise ECG Recording, D. Barschdorff, M. Erig, Journal of Biomedical Engineering 43, pp. 34 - 39 (1998)

4. HeikoGesche • DetlefGrosskurth • GertKu"chler • Andreas Patzak "Continuous blood pressure measurement by using the pulse transit time: comparison to a cuff-based method", Eur J ApplPhysiol DOI 10.1007/s00421-0111983-3, Springer.

5. Clinical Evaluation of Continuous Noninvasive Blood Pressure Monitoring: Accuracy and Tracking Capabilities, C. C. Young, J. B. Mark, W. White, A. DeBree, J. S. Vender, A. Fleming, Journal of Clinical Monitoring 11, pp. $245-252$ (1995) 